Potravinarstvo Slovak Journal of Food Sciences

vol. 15, 2021, p. 627-631

https://doi.org/10.5219/1664

Received: 23 April 2021. Accepted: 6 June 2021.

Available online: 28 July 2021 at www.potravinarstvo.com

(C) 2021 Potravinarstvo Slovak Journal of Food Sciences, License: CC BY 4.0

ISSN 1337-0960 (online)

\title{
GENETIC IDENTIFICATION OF THE CAUSATIVE AGENT OF BRUCELLOSIS
}

\author{
Yerkebulan Jakipov, Muafik Mustafin, Anda Valdovska, Sayat Baiseitov, Ayauly Aitkulova
}

\begin{abstract}
The development of animal husbandry suffers various kinds of losses due to the spread of infectious diseases among animals, in particular Brucellosis. A challenge faced by Brucella researchers has been the accurate identification of new isolates within the genus while preserving sufficient, and not excessive, biosafety and biosecurity requirements. The availability of discriminatory molecular typing tools to inform and assist conventional epidemiological approaches would be invaluable in controlling these infections, but efforts have been hampered by the genetic homogeneity of the genus. In this work, for better identification of infection, for control and monitor the source of outbreaks in prosperous areas was carried out identification of Brucella spp. strains which circulating in the Kostanay region. For this was used using multilocus analysis of a variable number of tandem repeats sequenced by $16 \mathrm{~s}-$ PNK on a genetic analyzer (sequencer). According to the results of a study of cattle, cultures of microorganisms were infected: No. 4, 5, 7, 8. Comparison of the obtained results with similar results of domestic and foreign works by A. Shevtsov, G. Borrello, P. Le Fleche, G. Garofolo suggest that the genotyping of local strains has an importance in the molecular epizootology of the Republic of Kazakhstan.
\end{abstract}

Keywords: amplification; sequencing; Brucella abortus; Brucella melitensis; strain

\section{INTRODUCTION}

Brucellosis is a zoonotic disease of major public health, animal welfare, and economic significance worldwide. In humans, infection with Brucella can lead to a chronic debilitating infection; in domesticated animals, the main symptom is a reproductive failure. Among the 12 species currently proposed in this genus, Brucella melitensis, Brucella abortus, and Brucella suis are highly dangerous for humans and cause disease with severe complications and chronic processes (Meyer, 1990; Godfroid et al., 2011). Rare cases of infection of people with other Brucella species are also recorded (Ficht, 2010). Despite its low mortality rates, brucellosis is a very important public health problem in Kazakhstan. Brucellosis causes great economic damage to livestock. The disease is accompanied by mass abortions and barrenness of animals suffering from brucellosis, joint damage, inflammation of mucous bags (in horses at the withers and the nape of the neck), the sex glands, the appearance of abscesses (more often in pigs), a decrease in the viability of offspring, a decrease in animal productivity. At the same time, elimination of brucellosis requires great effort and cost (Demirev, 2013; Whatmore et al., 2006; Whatmore, 2009). The development of discriminatory molecular tools for the identification and typing of Brucella has been problematic, reflecting the lack of genetic polymorphism in Brucella.

To address the issue of epizootology and understanding the pathogenesis of the disease, an accurate and timely diagnosis is necessary. The success of the fight against brucellosis depends on the rate of detection of all infected animals, on the effectiveness of diagnostic studies. The traditional system of identification and systematization of isolated field strains of Brucella is imperfect. None of the existing molecular tools provide adequate resolution to confidently permit epidemiological traceback in the case of accidental import or deliberate release. The phenotypic methods used allow differentiation of the causative agent of brucellosis to the species-specific level, but they are characterized by laboriousness, duration, and nonspecificity of the analysis (Whatmore, 2009; Alton et al., 1988; Le Flèche et al., 2006). These methods do not meet modern typing requirements. The solution to this problem is seen in the use of modern methods of molecular genetic typing of Brucella isolates.

Using molecular methods of analysis, genomic polymorphism is simply detected - quantitative changes in mini- and microsatellite DNA sequences (decrease or increase in the number of repeats), creating a series of unique alleles for each variable locus. These types of polymorphic loci are convenient markers. The phenomenon of polymorphism underlies the common method of identifying mutant DNA fragments - analysis of microsatellite markers (Ayala and Kiger, 1980; Gershkovich, 1968; Al Dahouk et al., 2007, Kattar et al., 2008). In this regard, the use of information about the causative agent of brucellosis obtained in the analysis of multilocus provides high-quality, effective elimination and prevention of the infectious process. 


\section{Scientific Hypothesis}

Using multilocus analysis with a variable number of tandem to create a molecular genetic cluster in the $16 \mathrm{~S}$ rRNA epizootic. Recursive multistep analysis of a tandem of a molecular genetic cluster in epizootology using $16 \mathrm{~S}$ rRNA in the Kostanay region strain of genotyping. In addition, the data obtained can be used for epidemiological surveillance and rapid detection of foci of brucellosis in epidemic zones. Currently, the research of the genetic modification of Brucella through 16s rRNA is necessary for a better understanding of the spread and monitoring of the occurrence of this infection in these areas.

\section{MATERIAL AND METHODOLOGY \\ Samples}

An object of study - cultures of microorganisms: No. 4, 5, 7,8 . The subject of the study is the polymorphism of a fragment of ribosomal RNA (16S rRNA). The study material - samples of genomic DNA isolated from the obtained cultures of microorganisms.

\section{Instruments}

Capillary genetic analyzer 8-16s rrna 3500 genetic Analyzer ("Applied Biosystems", USA) for genotyping and defining Brucella isolators is a worthy tool for identifying genotypes.

\section{Laboratory Methods}

On the initial stages of the work, the following processes took place:

1. Isolation of DNA from microorganism cultures. For DNA extraction, the commercial CTAB Sample Kit was used (DNA-Technology, Russia). The work with the kit was carried out following the manufacturer's instructions.

2. Determination of DNA nucleotide sequence by the method of F. Sanger (enzymatic method)

3. Sequencing steps. Sequencing of amplification fragments was performed using the ABI PRISM Big Dye Terminator v.3.1 kit (Applied Biosystems, USA), according to the manufacturer's instructions using the Applied Biosystems 3500 Genetic Analyzer 8 capillary genetic analyzer (Applied Biosystems, USA). After conducting electrophoretic analysis of amplification 2 using 2\% agarose, we placed micro schemes with amplification products as a tripod. Each row of gel tracks must have a DNA molecular weight marker (Ferreira et al., 2012). Next on paraffin paper with a pipette dosing, we add $10 \mu \mathrm{L}$ of amplification, $2 \mu \mathrm{L}$ of 6X DNA Loading Dye, pipetting. $10 \mu \mathrm{L}$ is poured into the well of the agarose gel. Electrophoresis results were registered using a QUANTUM Model 1100 SUPER Documentation System Gel, which has ultra-high sensitivity, including for fluorescence, is controlled via PC. One band is visible on the resulting image, which indicates the purity of the obtained cultures, the presence of two or more bands in the results indicates that the cultures are contaminated by other microorganisms (Corbel, 1997; Gandara et al., 2001). In this case, it is impossible to conduct sequencing of this culture (Whatmore et al., 2006, Gwida et al., 2012).

4. Clearing the amplification from residual reagents with CleanSweep reagent, mix composition. Incubated at $37^{\circ} \mathrm{C}$ $-15 \mathrm{~min}, 80^{\circ} \mathrm{C}-15 \mathrm{~min}$ and left for storage.

\section{Sample preparation}

Cultures were obtained in the Kostanay regional branch of RSE on REM "Republican veterinary laboratory".
Microorganisms had been seeded on a specific nutrient medium for Brucella - erythritol agar had been grown for $20-30$ days at $37-38{ }^{\circ} \mathrm{C}$. For microscopic examination, staining was performed according to Shuliak-Shin.

\section{Statistical Analysis}

Sequence data were obtained by overlaying the sequenced sequences from the forward and reverse primers using the SeqMan program (Lasergene 6). Alignment was performed using the on-line BLAST program hosted on the NCBI website - National Center for Biotechnological Information, USA.

\section{RESULTS AND DISCUSSION}

Since there were essentially no molecular tools that could provide useful discrimination beyond the level of biovar, the first aim of this study was to characterize highly discriminatory loci that would facilitate the development of tools to permit epidemiological traceback of Brucella to the source of infection The initial stages of the work focused on: 1. Isolation of DNA from microorganism cultures; 2. Determination of DNA nucleotide sequence; 3. Sequencing steps; 4. Cleared the amplification from residual reagents with CleanSweep reagent, mix composition (Table 1).

Then a sequencing reaction was performed. Each PCR product was pipetted into the 2 wells of a strip of $1 \mu \mathrm{L}$ (the primers were excavated separately in the wells, to the first was added straight, to the second - the reverse) + the reaction mixture of the following composition (Table 2).

Put on the amplification according to the following program (Table 3). The rate of increase or decrease in temperature $-1^{\circ} \mathrm{C} / \mathrm{sec}$.

Purification (precipitation) before sequencing (performed in a plate with cooling) was performed.

Table 1 The composition of the mixture for the purification of amplification products.

\begin{tabular}{ll}
\hline Mix component & Amount per reaction \\
\hline CleanSweep & $2 \mu \mathrm{L}$ \\
Amplification & $5 \mu \mathrm{L}$ \\
\hline
\end{tabular}

Table 2 The reaction mixture for sequencing.

\begin{tabular}{ll}
\hline Component of the reaction mixture & $\begin{array}{l}\text { Amount per } \\
\text { reaction }\end{array}$ \\
\hline $\begin{array}{l}\text { Primer 16S-F (ITS-F), } \\
\text { either primer 16S-R (ITS-R) }\end{array}$ & $1 \mu \mathrm{L}$ \\
Purified amplification & $1,5 \mu \mathrm{L}$ \\
Big Dye Terminator 3.1 Ready Reaction & $4 \mu \mathrm{L}$ \\
Mix & $4 \mu \mathrm{L}$ \\
\hline Deionized water & \\
\hline
\end{tabular}

Table 3 Amplification program.

\begin{tabular}{lll}
\hline Temperature & Time & Number of cycles \\
\hline $96^{\circ} \mathrm{C}$ & $1 \mathrm{~min}$ & \\
$96^{\circ} \mathrm{C}$ & $10 \mathrm{sec}$ & \\
$50^{\circ} \mathrm{C}$ & $5 \mathrm{sec}$ & $25 \mathrm{cycles}$ \\
$60^{\circ} \mathrm{C}$ & $1,5 \mathrm{~min}$ & \\
$4^{\circ} \mathrm{C}$ & storage & \\
\hline
\end{tabular}


$45 \mu \mathrm{L}$ Sam Solution and $10 \mu \mathrm{L}$ Xterminator Solution had been added to the amplification obtained after the sequential reaction, shaking the reagent strongly, as it quickly precipitated. The resulting mixture was vortexed at $1800 \mathrm{rpm}$ for 20 minutes using Digital Vortex-Genie 2. After that, the plate was centrifuged at $1000 \mathrm{~g}$ for 2 minutes.
$15 \mu \mathrm{L}$ of the supernatant was collected and the plate was loaded into the sequencer. As a result of sequencing the cultures of microorganisms, the following results were obtained (Table 4, Figure 1).

Table 4 Results of identification by the method of analysis of the nucleotide sequence of the gene 16S rRNA.

The name of
the strain

The sequence of the fragment $16 \mathrm{~S}$ r RNA

\section{Identification of nucleotide \\ sequences in the international database \\ (http://www.ncbi.nlm.nih.gov/) BLAST algorithm}

The name of the matches
strain

GTATCAAAGGCAGTTCCGGGGTTGAGCCCCGGGATTTCACCCCTGACT TAAAAGTCCGCCTACGTGCGCTTTACGCCCAGTAAATCCGAACAACG 1/8 CTAGCCCCCTTCGTATTACCGCGGCTGCTGGCACGAAGTTAGCCGGGG CTTCTTCTCCGGTTACCGTCATTATCTTCACCGGTGAAAGAGCTTTACA ACCCTAGGGCCTTCATCACTCACGCGGCATGGCTGGATCAGGCTTGCG CCCATTGTCCAATATTCCCCACTGCTGCCTCCCGTAA

TCCGGGGTTGAGCCCCGGGATTTCACCCCTGACTTAAAAGTCCGCCTA CGTGCGCTTTACGCCCAGTAAATCCGAACAACGCTAGCCCCCTTCGTA TTACCGCGGCTGCTGGCACGAAGTTAGCCGGGGCTTCTTCTCCGGTTA CCGTCATTATCTTCACCGGTGAAAGAGCTTTACAACCCTAGGGCCTTC ATCACTCACGCGGCATGGCTGGATCAGGCTTGCGCCCATTGTCCAATA TTCCCCACTGCTGCCTCCCGTA

AGTTCCGGGGTTGAGCCCCGGGATTTCACCCCTGACTTAAAAGTCCGC CTACGTGCGCTTTACGCCCAGTAAATCCGAACAACGCTAGCCCCCTTC GTATTACCGCGGCTGCTGGCACGAAGTTAGCCGGGGCTTCTTCTCCGG TTACCGTCATTATCTTCACCGGTGAAAGAGCTTTACAACCCTAGGGCC TTCATCACTCACGCGGCATGGCTGGATCAGGCTTGCGCCCATTGTCCA ATATTCCCCACTGCTGCCTC

ACCTCTACACTCGGAATTCCACTCACСTCTACCATACTCAAGACTTCC AGTATCAAAGGCAGTTCCGGGGTTGAGCCCCGGGATTTCACCCCTGA CTTAAAAGTCCGCCTACGTGCGCTTTACGCCCAGTAAATCCGAACAAC $5 / 5 \mathrm{M}$ GCTAGCCCCCTTCGTATTACCGCGGCTGCTGGCACGAAGTTAGCCGGG GCTTCTTCTCCGGTTACCGTCATTATCTTCACCGGTGAAAGAGCTTTAC AACCCTAGGGCCTTCATCACTCACGCGGCATGGCTGGATCAGGCTTGC GCCCATTGTCCAATATTCCCCACTGCTGCCTCCCGTAA

GGGGCTAGCGTTGTTCGGATTTACTGGGCGTAAAGCGCACGTAGGCG GACTTTTAAGTCAGGGGTGAAATCCCGGGGCTCAACCCCGGAACTGC CTTTGATACTGGAAGTCTTGAGTATGGTAGAGGTGAGTGGAATTCCGA GTGTAGAGGTGAAATTCGTAGATATTCGGAGGAACACCAGTGGCGAA GGCGGCTCACTGGACCATTACTGACGCTGAGGTGCGAAAGCGTGGGG AGCAAACAGGATTAGATACCCTGGTA

AGGGGGCTAGCGTTGTTCGGATTTACTGGGCGTAAAGCGCACGTAGG CGGACTTTTAAGTCAGGGGTGAAATCCCGGGGCTCAACCCCGGAACT

7/5 GCCTTTGATACTGGAAGTCTTGAGTATGGTAGAGGTGAGTGGAATTCC GAGTGTAGAGGTGAAATTCGTAGATATTCGGAGGAACACCAGTGGCG AAGGCGGCTCACTGGACCATTACTGACGCTGAGGTGCGAAAGCGTGG GGAGCAAACAGGATTAGATACCCTGGTAGA

CTTCCAGTATCAAAGGCAGTTCCGGGGTTGAGCCCCGGGATTTCACCC CTGACTTAAAAGTCCGCCTACGTGCGCTTTACGCCCAGTAAATCCGAA

8/5 CAACGCTAGCCCCCTTCGTATTACCGCGGCTGCTGGCACGAAGTTAGC CGGGGCTTCTTCTCCGGTTACCGTCATTATCTTCACCGGTGAAAGAGC TTTACAACCCTAGGGCCTTCATCACTCACGCGGCATGGCTGGATCAGG CTTGCGCCCATTGTCCAATATTCCCCACTGCTGCCTCCCGTAA ACCTCTACACTCGGAATTCCACTCACCTCTACCATACTCAAGACTTCC AGTATCAAAGGCAGTTCCGGGGTTGAGCCCCGGGATTTCACCCCTGA CTTAAAAGTCCGCCTACGTGCGCTTTACGCCCAGTAAATCCGAACAAC 9/7 GCTAGCCCCCTTCGTATTACCGCGGCTGCTGGCACGAAGTTAGCCGGG GCTTCTTCTCCGGTTACCGTCATTATCTTCACCGGTGAAAGAGCTTTAC AACCCTAGGGCCTTCATCACTCACGCGGCATGGCTGGATCAGGCTTGC GCCCATTGTCCAATATTCCCCACTGCTGCCTCCCGTAA

AAGGCAGTTCCGGGGTTGAGCCCCGGGATTTCACCCCTGACTTAAAA GTCCGCCTACGTGCGCTTTACGCCCAGTAAATCCGAACAACGCTAGCC CCCTTCGTATTACCGCGGCTGCTGGCACGAAGTTAGCCGGGGCTTCTT CTCCGGTTACCGTCATTATCTTCACCGGTGAAAGAGCTTTACAACCCT AGGGCCTTCATCACTCACGCGGCATGGCTGGATCAGGCTTGCGCCCAT TGTCCAATATTCCCCACTGCTGCCTCCCGTAA
Brucella abortus

$100 \%$

Brucella melitensis

$100 \%$

Brucella canis $100 \%$

Brucella abortus $100 \%$

Brucella melitensis $100 \%$

Brucella canis $100 \%$

Brucella abortus $100 \%$

Brucella melitensis $100 \%$

Brucella canis $100 \%$

Brucella abortus $100 \%$

Brucella melitensis $100 \%$

Brucella canis $100 \%$

Brucella abortus $100 \%$

Brucella melitensis $100 \%$

Brucella canis $100 \%$

Brucella abortus $100 \%$

Brucella melitensis $100 \%$

Brucella canis $100 \%$

Brucella abortus $100 \%$

Brucella melitensis $100 \%$

Brucella canis $100 \%$

Brucella abortus $100 \%$

Brucella melitensis $100 \%$

Brucella canis $100 \%$

Brucella abortus $100 \%$

Brucella melitensis $100 \%$

Brucella canis $100 \%$ 


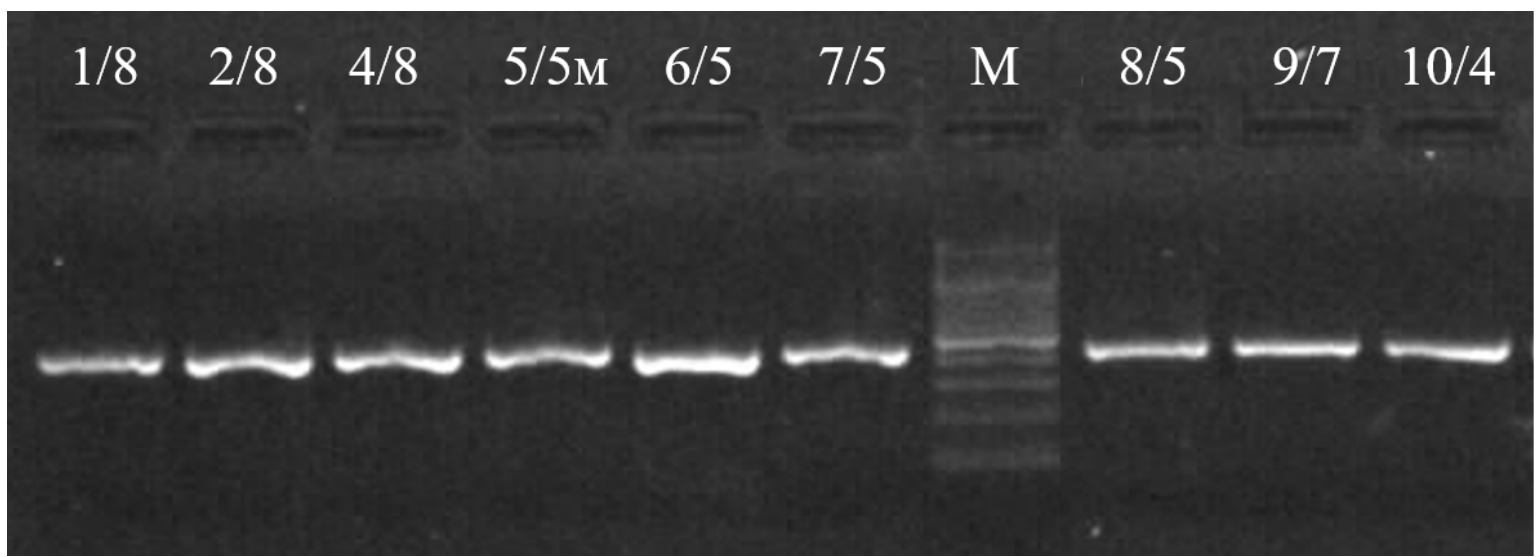

Figure 1 Electrophoregram of PCR products with primers for 16S rRNA (M - molecular weight marker 100 bp.

The success of brucellosis control depends on the speed of detection of all infected animals and the effectiveness of diagnostic tests (Alton et al., 1988; Meyer, 1990; Yagupsky, 1999). Up-to-date disease detection helps to take steps to isolate the infected animal early as a source of infection (Bricker and Ewalt, 2005; Ridler et al., 2005). The following serological tests for animal brucellosis are officially recognized in Kazakhstan: RBS, AR, CBD, and ELISA. The rate of serological reactions in brucellosis is unstable (Ficht, 2010; Scholz and Vergnaud, 2013). While each method is complex with other methods, it does not isolate all sick animals. Traditional phenotypic differentiation methods make it difficult to standardize the situation, which is the reason for erroneous differentiation (Pappas et al., 2006; Godfroid et al., 2011, Borriello et al., 2013). It is known that the phenotypic characteristics of bacteria can vary depending on the growing conditions and the allelic state responsible for gene expression. The most conservative structure that characterizes the type of microorganism in its gene (Ayala and Kiger, 1980; Gershkovich, 1968; Al Dahouk et al., 2007, Kattar et al., 2008). In this regard, the most promising is the assessment based on the nature of the pathogen genome.

The solution of the problem made it possible to determine the genotype of the studied strains using cluster analysis and to start an investigation of the outbreak in the regions endemic for brucellosis (Frachetti et al., 2017). Timely provision of information on the causative agent of brucellosis, obtained using multilocus analysis, for the use of scientific research results in veterinary practice, which ensures high-quality monitoring of the epidemic process, effective eradication, and prevention (Le Flèche et al., 2006; Whatmore, 2009; Demirev, 2013). The expediency of the introduction of 16S rRNA is an accurate and timely diagnosis of brucellosis in animals since the timely detection of the disease will help to take measures for the early isolation of the animal as a source of infection and prevent its further development and spread (Garofolo et al., 2013; Shevtsov et al., 2015).

\section{CONCLUSION}

The region of the 16S rRNA gene sequence among the primers used helps to determine $100 \%$ affinity, but the specificity is not enough to identify the species. The obtained data are consistent with bacteriological data (colony shape - S, bluish-transparent in daylight; microscopy: round negative rods with rounded ends, located immediately; all characteristics correspond to Brucella abortus). It can be said that the Brucella family is present in the studied samples of microorganisms (no. 4, 5, 7, 8). It turned out that the study of the genetic diversity of brucellosis using Brucella spp. The genetic structure of the Kazakh population has not been studied enough.

Multiple-locus analysis genotyping constitutes a convenient medium-resolution classification assay for large-scale investigations. Follow-up studies based on the whole genome sequencing of selected strains will be necessary to more precisely decipher the dynamics of strain circulation both within Kazakhstan and with Kazakhstan's neighboring countries. Such studies will also permit to design of new low-cost genotyping assays tailored for Central Asia.

\section{REFERENCES}

Al Dahouk, S., Le Flèche, P., Nöckler, K., Jacques, I., Grayon, M., Scholz, H. C., Tomaso, H., Vergnaud, G., Neubauer, H. 2007. Evaluation of Brucella MLVA typing for human brucellosis. Journal of Microbiological Methods, vol. 69, no. $1, \quad$ p. $137-145$. https://doi.org/10.1016/j.mimet.2006.12.015

Alton, G. G., Jones, L. M., Angus, R. D., Verger, J. M. 1988. Techniques for the brucellosis laboratory. Paris, France : INRA, 190 p. ISBN 2738000428.

Ayala, F. J., Kiger, J. A. 1980. Modern Genetics. San Mateo, USA : Benjamin/Cummings, 89 p. ISBN 0805303162.

Borriello, G., Peletto, S., Lucibelli, M. G., Acutis, P. L., Ercolini, D., Galiero, G. 2013. Link between geographical origin and occurrence of Brucella abortus biovars in cow and water buffalo herds. Applied and Environmental Microbiology, vol. 79, no. 3, p. 1039-1043. https://doi.org/10.1128/AEM.02887-12

Bricker, B. J., Ewalt, D. R. 2005. Evaluation of the HOOFPrint assay for typing Brucella abortus strains isolated from cattle in the United States: results with four performance criteria. BMC Microbiology, vol. 5, p. 37 https://doi.org/10.1186\%2F1471-2180-5-37

Corbel, M. J. 1997. Brucellosis: an overview. Emerging Infectious Diseases, vol. 3, no. 2, p. 213-221 https://dx.doi.org/10.3201\%2Feid0302.970219

Demirev, P. A. 2013. Dried blood spots: Analysis and applications. Analytical Chemistry, vol. 85 no. 2, p. 779-789. https://doi.org/10.1021/ac303205m

Ferreira, A. C., Chambel, L., Tenreiro, T., Cardoso, R., Flor, L., Dias, I. T., Pacheco, T., Garin-Bastuji, B., Le Fléche, P., Vergnaud, G., Tenreiro, R., de Sá, M. I. C. 2012. MLVA16 
typing of portuguese human and animal Brucella melitensis and Brucella abortus isolates. PLoS One vol. 7, no. 8, p. e42514. https://doi.org/10.1371/journal.pone.0042514

Ficht, T. 2010. Brucella taxonomy and evolution. Future Microbiology, vol. 5, no. 6, p. 859-866. https://doi.org/10.2217/fmb.10.52

Frachetti, M. D., Smith, C. E., Traub, C. M., Williams, T. 2017. Nomadic ecology shaped the highland geography of Asia's silk roads. Nature, vol. 543, p. 193-198. https://doi.org/10.1038/nature21696

Gandara, B., Merino, A. L., Rogel, M. A., MartinezRomero, E. 2001. Limited genetic diversity of Brucella spp. Journal of Clinical Microbiology, vol. 39, no. 1, p. 235-240. https://doi.org/10.1128\%2FJCM.39.1.235-240.2001

Garofolo, G., Di Giannatale, E., De Massis, F., Zilli, K., Ancora, M., Cammà, C., Calistri, P., Foster, J. T. 2013. Investigating genetic diversity of Brucella abortus and Brucella melitensis in Italy with MLVA-16. Infection, Genetics and Evolution, vol. $19, \quad$ p. $\quad$ 59-70. https://doi.org/10.1016/j.meegid.2013.06.021

Gershkovich, I. 1968. Genetics. Moscow, USSR : Nauka, $698 \mathrm{p}$.

Godfroid, J., Scholz, H. C., Barbier, T., Nicolas, C., Wattiau, P., Fretin, D., Whatmore, A. M., Cloeckaert, A., Blasco, J. M., Moriyon, I., Saegerman, C., Muma, J. B., Dahouk, S. Al. Neubauer, H., Letesson, J.-J. 2011. Brucellosis at the animal/ecosystem/human interface at the beginning of the 21st century. Preventive Veterinary Medicine, vol. 102, no. 2, p. 118-131. https://doi.org/10.1016/j.prevetmed.2011.04.007

Gwida, M., Neubauer, H., Ilhan, Z., Schmoock, G., Melzer, F., Nöckler, K., Janczyk, P., Tomaso, H., Rösler, U., Dahouk, S. Al. 2012. Cross-border molecular tracing of brucellosis in Europe. Comparative Immunology, Microbiology and Infectious Diseases, vol. 35, no. 2, p. 181-185. https://doi.org/10.1016/j.cimid.2011.12.012

Kattar, M. M., Jaafar, R. F., Araj, G. F., Le Flèche, P., Matar, G. M., Rached, R. A., Khalife, S., Vergnaud, G. 2008. Evaluation of a multilocus variable-number tandem-repeat analysis scheme for typing human brucella isolates in a region of brucellosis endemicity. Journal of Clinical Microbiology, vol. 46, no. 12, p. 3935-3940. https://doi.org/10.1128/JCM.00464-08

Le Flèche, P., Jacques, I., Grayon, M., Al Dahouk, S., Bouchon, P., Denoeud, F., Nöckler, K., Neubauer, H., Guilloteau, L. A., Vergnaud, G. 2006. Evaluation and selection of tandem repeat loci for a Brucella MLVA typing assay. BMC Microbiology, vol. 6, no. 1, p. 9. https://doi.org/10.1186/14712180-6-9

Meyer, M. E. 1990. Evolutionary development and taxonomy of the genus Brucella. In Adams, L. G. Advances in Brucellosis Research. Galveston, USA : Texas A\&M University Press, 542 p. ISBN-13 978-0-89096-447-7.

Pappas, G., Panagopoulou, P., Christou, L., Akritidis, N. 2006. Brucella as a biological weapon. Cellular and Molecular Life Sciences, vol. 63, p. 2229-2236. https://doi.org/10.1007/s00018-006-6311-6314

Ridler, A. L., Leyland, M. J., Fenwick, S. G., West, D. M. 2005. Demonstration of polymorphism among Brucella ovis field isolates by pulsed-field gel electrophoresis. Veterinary Microbiology, vol. 108, no. 1-2, p. 69-74. https://doi.org/10.1016/j.vetmic.2005.02.004

Shevtsov, A., Ramanculov, E., Shevtsova, E., Kairzhanova, A., Tarlykov, P., Filipenko, M., Dymova, M., Abisheva, G., Jailbekova, A., Kamalova, D., Chsherbakov, A., Tulegenov, S., Akhmetova, A., Sytnik, I., Karibaev, T., Mukanov, K. 2015.
Genetic diversity of Brucella abortus and Brucella melitensis in Kazakhstan using MLVA-16. Infection, Genetics and Evolution, vol. 34, $\quad$ p. $173-180$. https://doi.org/10.1016/j.meegid.2015.07.008

Scholz, H. C., Vergnaud, G. 2013. Molecular characterisation of Brucella species. Revue Scientifique et Technique, vol. 32, no. 1, p. 149-162. https://doi.org/10.20506/rst.32.1.2189

Whatmore, A. M. 2009. Current understanding of the genetic diversity of Brucella, an expanding genus of zoonotic pathogens. Infection, Genetics and Evolution, vol. 9, no. 6, p. 1168-1184. https://doi.org/10.1016/j.meegid.2009.07.001

Whatmore, A. M., Shankster, S. J., Perrett, L. L. Murphy, T. J., Brew, S. D., Thirlwall, R. E., Cutler, S. J., MacMillan, A. P. 2006. Identification and characterization of variable-number tandem-repeat markers for typing of Brucella spp. Journal of Clinical Microbiology, vol. 44, no. 6, p. 1982-1993. https://doi.org/10.1128/jcm.02039-05

Yagupsky, P. 1999. Detection of Brucellae in blood cultures. Journal of Clinical Microbiology, vol. 37, p. 3437-3442. https://doi.org/10.1128/jcm.37.11.3437-3442.1999

\section{Conflict of Interest:}

The authors declare no conflict of interest.

\section{Ethical Statement:}

This article does not contain any studies that would require an ethical statement.

\section{Contact Address:}

*Yerkebulan Jakipov, Akhmet Baitursynov Kostanay State University, Department of Veterinary Medicine, Baytursynov Str., 47A, 110000, Kostanay, Republic of Kazakhstan, Tel.: +7 (7142) 558463,

E-mail: jakipov6056@nuos.pro

ORCID: https://orcid.org/0000-0002-9747-9411

Muafik Mustafin, Akhmet Baitursynov Kostanay State University, Department of Veterinary Medicine, Baytursynov Str., 47A, 110000, Kostanay, Republic of Kazakhstan, Tel.: +7 (7142) 558488,

E-mail: mmustafin@tanu.pro

ORCID: https://orcid.org/0000-0003-0720-3719

Anda Valdovska, Latvia University of Life Sciences and Technologies, Faculty of Veterinary Medicine, Liela Str., 2, LV-3001, Jelgava, Latvia, Tel.: +371 630 24662,

E-mail: valdovska65@politechnika.pro

ORCID: https://orcid.org/0000-0003-2044-5042

Sayat Baiseitov, Omsk State Agrarian University named after P.A. Stolypin, Department of Veterinary Microbiology, Infectious and Invasive Diseases, Institutskaya Sq., 1, 644008, Omsk, Russian Federation, Tel.: +7 (3812) 25-05-70,

E-mail: s.baiseitov@tanu.pro

ORCID: https://orcid.org/0000-0001-7252-9980

Ayauly Aitkulova, Kazakh National Agrarian University, Department of Biological Safety, Abai Ave., 8, 050010, Almaty, Republic of Kazakhstan, Tel.: +7 (727) 261-5857, E-mail: aaitkulova@nuos.pro ORCID: https://orcid.org/0000-0003-0900-9864

Corresponding author: * 